\title{
Exanguíneo-transfusión en Coma Hepático
}

\author{
Dres.: César Cáceres," Patricio Herrera, Isidoro \\ Horwitz," Osvaldo Danús."
}

Desde que Lee y Tink en 1958 (I) comunicaron la recuperación de un paciente en cona hepático post hepatitis viral, utilizando como procedimiento terapéutico la exanguíneo transfusión, ha aparecido un gran número de publicaciones informando de este procedimiento y sus resultados $(2,14)$. Pese a haberse ensayado en un importante número de casos, la impresión optimista inicial ha derivado hacia la incertidumbre actual sobre la utilidad real de este procedimiento complejo y costoso.

En el Departamento de Pediatría del Hos. pital Roberto del Río se han tratado ya, desde 1966, 37 casos de insuficiencia hepática grave con exanguíneo transfusión, de los cuales 10 fueron dados a conocer en una publicación inicial (4); el material de la presente comunicación da cuenta de algunos aspectos sobre estos enfermos y el procedimiento en cuestión, analizándose en conjunto el grupo de los 37 pacientes.

\section{MATERIAL Y METODO}

E1 material está constituido por 37 niños que padecían de insuficiencia hepática post hepatitis viral entre los años 1966 y 1975. En todos los pacientes se diagnosticó hepatitis viral, probablemente por virus $A$, de acuerdo con los antecedentes clínicos y de laboratorio usuales. El coma hepático fue definido clinicamente en base al criterio de Adams y

\footnotetext{
-Departamento de Pediatria, Hospital Roberto
} del Ría.
Foley $(2,15,17)$; se analizó además el resurtado de los siguientes exámenes de Iaboratorio: transaminasas, protrombina, electrolitograma, glutamina en LCR, bilirrubina.

Todos los pacientes recibieron tratamiento médico consistente en el suministro intravenoso de dextrosa, corrección de desequilibrios hidroelectrolítico y ácido básico, restricción de proteínas en la dieta, administración oral de soluciones de hidratos de carbono, neomicina, enema evacuante, vitamina $\mathrm{K}$ intramuscular y corticoesteroides (16). Cuando utilizando el tratamiento recién descrito no se logró obtener respuesta favorable (regresión de las condiciones clínicas en un período de 12 horas) se recurrió, a la exanguí. neo transfusión. Dicho procedimiento se practicó con dos volemias de sangre heparinizada, por cateterización de la vena safena.

La edad de los pacientes fluctuó entre 1 mes y 12 años, constituyendo el grupo menor de 4 años el 74\% del grupo (Tabla № 2). En relación al sexo, 19 pacientes pertenecían al sexo masculino y 18 al sexo femenino (Tablas No 1 y 2).

\section{RESULTADOS}

En el momento de ingreso a la unidad de hospitalización, sólo 6 de los 37 niños no presentaban encefalopatía, pero evolucionan en tiempos variables hacia esta complicacion de modo que en el momento de efectuar la primera exanguíneo transfusión, un paciente presentaba encefalopatía grado I; 5 grado II; 18 grado III y 13 encefalopatía grado IV. 
En general estos pacientes recibieron entre uno y once recambios sanguíneos con el siguiente resultado (Tabla $\mathrm{N}^{\circ} 3$ ):

El niño con cncefalopatía grado I mejoró después de 3 exanguíneo transfusiones; de los 5 niños que presentaban grado IJ: 2 mejoran y tres fallecen, habiendo recibido entre 2 y 3 exanguíneo transfusiones. Del grupo con grado III fallecen 12 , sobreviven 6 y se les practicó el procedimiento entre 1 y 6 veces; $y$, del grupo grado IV fallecen 9 y sobreviven 4 , habiendo recibido entre una $y$ once exanguíneo transfusiones.

La tabla $N^{\circ} 4$ muestra la letalidad por grupo etario no apreciándose variaciones significativas; en efecto, de 18 pacientes menores de 2 años fallecen $11(61,11 \%)$; de 15 niños entre 3 y 6 años de edad fallecen 10 $(66,66 \%)$, y de 4 mayores de 6 años fallecen $3(75 \%)$. La letalidad global del grupo fue de 24 pacientes $(64,84 \%)$.

Tampoco se aprecia variación de la letalidad en relación al período previo de enfermedad ya que de 21 niños, cuya enfermedad previa al ingreso era menor de 14 días, fallecen $14(66,66 \%)$, y de 16 niños con más de 14 días previos de enfermedad, fallecen 10 (62,5\%) (Tabla No 5 ).

Se analizó además la relación existente entre cifras de protrombinemia y bilirrubina al ingreso y letalidad.

En la Tabla № 6 se aprecia la relación de los valores de protrombina con la sobrevida, encontrándose que cuando la protrombina era infetior a $20 \%$, de un total de 26 pacientes sobreviven 7. En 7 casos la protrombina fluctuo entre 20 y $33 \%$, sobreviviendo 4 niños; respecto a la relación entre valores de bilirrubina y sobrevida (Tabla № 7 ), con valores de bilirrubinemia inferiores a $15 \mathrm{mg} . \%$ sobreviven 3 de 8 casos y con cifras superiores a $15 \mathrm{mg} . \%$ de 23 niños sobreviven 8 . El análisis estadístico de estos parámetros no revelo ningún valor significativo.

\section{COMENTARIO}

Aparentemente los resultados de la exangufneo transfusión desmejoran al incrementarse el número de casos sometidos al procedimiento; esto se advierte cuando se comparan la seric presentada por González y col. (4) con la experiencia actuat, teniendo la primera una sobrevida de $40 \%$ y ésta de un $33,33 \%$, que da un proncdio de $36,66 \%$ para el grupo total. Esto sugiere que el resultado obtenido no depende del procedjmiento, sino de factores de la enfermedad misma no predecibles o detectables con los criterios clínicos y de laboratorio vigentes, expresados como "potencial regenerativo hepático", inaccesible al estudio practicado. El escaso estudio necrópsico impide analizar causas de fracaso que no dependan de la insuficiencia hepática misma, pero puede atribuirse a ésta la muerte en la mayoría de los casos, ya que en los pacicntes en que esto fue posible (29\% de los fallecidos) no se detectó otra causa de muerte sino la insuficiencia hepática. La relativamente pequeña fracción de pacientes que sobrevivieron, tecomendaria ensayar el procedimiento mientras no existan razones suficientes para descartario o se establezca otro medio terapéutico más útil de recobrar estos enfermos. Mantenemos vigente la posibilidad de que el procedimiento empleado en etapas iniciales de encefalopatía (grado I a II) permitiria obtener mejores resultados.

\section{RESUMEN}

Se presenta la experiencia obtenida con el uso de exanguíneo transfusión en 37 niños con encefalopatia post hepatitis viral aguda. La edad de los niños fluctuó entre 1 mes y 12 aîos, constituyendo el grupo menor de 4 años el $74 \%$ del grupo. Del total de 37 niños recambiados fallecieron $24(64,85 \%)$. El análisis estadístico de algunos parámetros, entre ellos: edad de los pacientes, letalidad en relación a tiempo previo de evolución de la enfermedad, grado de encefalopatía al realizarse el recambio sanguíneo y cifras previas de protrombina y bilirrubina no mostró relación estadística significativa.

\section{SUMMARY}

An experiencie using exchange tranfusions in 37 children with acute viral post hepatitis encephalopathy is presented. 
The cbildren age oscillated between one month and 12 years, the $74 \%$ of the group were under 4 years.

From the 37 children 24 died $(64,85 \%)$. The statistical analysis of some parameters: patient's age, lethality related with the prior course of the disease, encephalopathy grade at the exchange, protrombin and bilirubin preliminary levels, didn't show any significant relation.

Tabla N: 1

\begin{abstract}
ALGUNAS CARACTERISTICAS CLINICAS Y DE LABORATORIO EN 37 NINTOS CON "COMA HEPATICO" POR HEPATITIS VIRAL
\end{abstract}

Hospital Roberto del Río - 1975

\begin{tabular}{|c|c|c|c|c|c|c|c|c|c|}
\hline $\begin{array}{l}\text { Caso } \\
N^{\circ}\end{array}$ & $\begin{array}{l}\text { Edad } \\
\text { Años }\end{array}$ & Sexo & $\begin{array}{l}\text { Grado de Coma } \\
\text { 1er. Recambio }\end{array}$ & $\begin{array}{l}\text { Ne de } \\
\text { Recambios }\end{array}$ & $\begin{array}{l}\text { Bil. } \\
\text { Total } \\
\text { mg. } \%\end{array}$ & $\begin{array}{c}\text { Protrom. } \\
\text { pre-Rec. } \\
\%\end{array}$ & $\begin{array}{l}\text { TGO } \\
\text { pre- } \\
\text { Rec. }\end{array}$ & $\begin{array}{l}\text { Glut. } \\
\text { LCR } \\
\text { mg.\% }\end{array}$ & Evolución \\
\hline 1 & 4 & $\mathbf{M}$ & 3 & 6 & 24 & 14 & 520 & - & $\mathbf{F}$ \\
\hline 2 & $3-1$ & $M$ & 4 & 1 & 28 & 10 & - & - & $\mathbf{F}$ \\
\hline 3 & 2 & $\mathrm{M}$ & 3 & 2 & 30 & 10 & 960 & - & $\mathbf{F}$ \\
\hline 4 & 2-1 & $\mathbf{M}$ & 4 & 2 & 35 & 14 & - & - & F \\
\hline 5 & 6 & F & 3 & 2 & 16 & 20 & 1.700 & $\longrightarrow$ & $\mathbf{F}$ \\
\hline 6 & 3 & F & 4 & 1 & 33 & 7 & 255 & - & $\mathbf{F}$ \\
\hline 7 & $6-9$ & $M$ & 4 & 2 & 11 & 5 & 1.500 & - & $\mathbf{v}$ \\
\hline 8 & $2-9$ & F & 4 & 2 & 27 & 10 & 385 & $\longrightarrow$ & $\mathbf{v}$ \\
\hline 9 & 1.8 & $F$ & 3 & 1 & 14 & 33 & 145 & - & $v$ \\
\hline 10 & 6 & $\mathbf{M}$ & 4 & 3 & 35 & 8 & 1.000 & 30 & $\mathbf{v}$ \\
\hline 11 & 24 & M & 3 & 2 & 28 & 6 & 750 & 21 & v \\
\hline 12 & 9 & $\mathbf{M}$ & 4 & 1 & 18 & 4 & 80 & 30 & $\mathbf{F}$ \\
\hline 13 & $1-3$ & $M$ & 4 & 1 & - & 0 & - & - & $\mathbf{F}$ \\
\hline 14 & 2 & $\mathbf{F}$ & 3 & 4 & 37 & 0 & 103 & 50 & F \\
\hline 15 & 12 & $\mathbf{M}$ & 4 & 2 & 24 & 26 & 555 & 22 & $\mathbf{F}$ \\
\hline 16 & $2-2$ & $\mathbf{F}$ & 2 & 2 & 28 & 8 & 1.500 & 33 & $\mathbf{F}$ \\
\hline 17 & $0-1$ & $\mathbf{F}$ & 2 & 2 & 17 & 18 & 210 & - & $\mathbf{F}$ \\
\hline 18 & $3-3$ & F & 4 & 1 & 13 & 0 & - & 16 & F \\
\hline 19 & $3-9$ & $\mathbf{M}$ & 2 & 2 & 15 & 24 & 1.000 & 12 & v \\
\hline 20 & 4.3 & $\mathbf{F}$ & 4 & 11 & 24 & 12 & 493 & 39 & V \\
\hline 21 & 1.6 & $\mathbf{P}$ & 1 & 3 & 27 & 8 & 750 & 16 & v \\
\hline 22 & $9-9$ & $\mathbf{M}$ & 3 & 1 & 18 & 4 & 96 & - & $\mathbf{F}$ \\
\hline 23 & 5 & $\mathbf{M}$ & 3 & 2 & 30 & 23 & 750 & - & v \\
\hline 24 & $1-9$ & $\mathbf{M}$ & 3 & 3 & 11 & 18 & - & 30 & $\mathbf{F}$ \\
\hline 25 & 1 & $\mathbf{F}$ & 3 & 3 & - & 15 & 750 & - & F \\
\hline 26 & 4 & $M$ & 4 & 1 & 28 & 14 & - & - & $\mathbf{F}$ \\
\hline 27 & $3-6$ & $\mathbf{F}$ & 3 & 1 & 12 & - & 750 & 62 & $\mathbf{F}$ \\
\hline 28 & 5 & $\mathbf{F}$ & 2 & 3 & 21 & 22 & 960 & - & $\mathbf{F}$ \\
\hline 29 & 1.9 & $\mathbf{M}$ & 3 & 1 & - & 0 & - & - & $\mathbf{F}$ \\
\hline 30 & 3 & $\mathbf{F}$ & 3 & 1 & 13 & 一 & - & سم & $\mathbf{p}$ \\
\hline 31 & 410 & $\mathbf{F}$ & 4 & 1 & 11 & 4 & 800 & - & $\mathbf{F}$ \\
\hline 32 & 0.2 & $\mathbf{F}$ & 3 & 2 & 17 & 18 & 260 & - & $\mathbf{F}$ \\
\hline 33 & $1-7$ & F & 3 & 4 & - & - & 750 & 75 & $\mathbf{V}$ \\
\hline 34 & 4 & M & 3 & 4 & - & - & 750 & - & V \\
\hline 35 & 2 & $\mathbf{F}$ & 3 & 3 & 17 & 14 & 750 & - & $\mathbf{F}$ \\
\hline 36 & $2-3$ & $\mathbf{M}$ & 2 & 2 & 10 & 23 & 750 & - & $\mathbf{V}$ \\
\hline 37 & $2 \cdot 5$ & $\mathbf{M}$ & 3 & 3 & 29 & 15 & 750 & 25 & $v$ \\
\hline
\end{tabular}


DISTRIBUCION POR EDAD EN 37 NINOS CON "COMA HEPATICO" POR HEPATITIS VIRAL

Hospital Roberto del Río - 1975

\begin{tabular}{lc}
\hline Edad en años & No de casos \\
\hline $0-2$ & 18 \\
$3-4$ & 11 \\
$5-6$ & 5 \\
$7-8$ & 0 \\
$9-10$ & 2 \\
Más de 10 & 1 \\
\hline Total & 37 \\
\hline
\end{tabular}

Tabla No 3

LETALIDAD EN RELACION AL GRADO DE "COMA HEPATICO" AL COMIENZO DEL TRATAMIENTO

Hospital Roberto del Río - 1975

\begin{tabular}{rrrrrr}
$\begin{array}{c}\text { Grado de } \\
\text { coma }\end{array}$ & No & No exanguine & \multicolumn{2}{c}{ Resultados } \\
nimonsfusiones & Mejoran & Fallecen \\
\hline I & 1 & 3 & & 1 & - \\
II & 5 & 2 a & 3 & 2 & 3 \\
III & 18 & 1 a & 6 & 6 & 12 \\
IV & 13 & 1 a 11 & 4 & 9 \\
\hline
\end{tabular}

Tabla N: 4

LETALIDAD EN RELACION A LA EDAD EN 37 NIÑOS CON "COMA HEPATICO"

Hospital Roberto del Rio - 1975

\begin{tabular}{lccc}
\hline $\begin{array}{c}\text { Edad en } \\
\text { años }\end{array}$ & $\begin{array}{c}\text { Total de } \\
\text { casos }\end{array}$ & $\begin{array}{c}\text { Ne de } \\
\text { fallecidos }\end{array}$ & $\begin{array}{c}\% \text { de } \\
\text { grupo }\end{array}$ \\
\hline 0 a 2 & 18 & 11 & 61,11 \\
3 a 6 & 15 & 10 & 66,66 \\
Mâs de 6 & 4 & 3 & 75 \\
\hline Total & 37 & 24 & \\
\hline
\end{tabular}

LETALIDAD EN RELACION AL PERIODO PREVIO DE ENFERMEDAD EN 37 NINOS CON "COMA HEPATICO"

Hospital Roberto del Río - 1975

\begin{tabular}{lccc}
\hline $\begin{array}{l}\text { Dias de } \\
\text { evolucion }\end{array}$ & $\begin{array}{c}\text { Total de } \\
\text { casos }\end{array}$ & $\begin{array}{c}\text { No de } \\
\text { fallecidos }\end{array}$ & $\begin{array}{c}\% \text { det } \\
\text { griapo }\end{array}$ \\
\hline Menos de 14 días & 21 & 14 & 66,66 \\
Más de 14 dias & 16 & 10 & 62,50 \\
\hline Total & 37 & 24 & \\
\hline
\end{tabular}

Tabla N: 6

LETALIDAD EN RELACION A CIFRA DE PROTROMBINEMIA AL INGRESO EN $3.3 \mathrm{NI}$. NOS CON "COMA HEPATICO"

Hospital Roberto del Río - 1975

\begin{tabular}{lccc}
\hline & \multicolumn{3}{c}{ Sobreviven } \\
Protrombinemia & Total & N: & $\%$ \\
\hline Menos de $20 \%$ & 26 & 7 & 26,9 \\
De 20 a $35 \%$ & 7 & 4 & 57,1 \\
\hline Total & 33 & 11 & 33,5 \\
\hline
\end{tabular}

Tabla № 7

LETALIDAD EN RELACION A CIFRA DE BILIRRUBINEMIA AL INGRESO EN 31 NINOS CON "COMA HEPATICO"

Flospital Roberto del Río - 1975

\begin{tabular}{lccc}
\hline & \multicolumn{3}{c}{ Sobreviven } \\
Bitirnubina & Total & No & $\%$ \\
\hline Menos de $15 \mathrm{mg} \%$ & 8 & 3 & 37,5 \\
$15 \mathrm{mg} \% \%$ o más & 23 & 8 & 34,8 \\
\hline Total & 31 & 11 & 35.5
\end{tabular}




\section{REFERENCIAS}

1. Lee C., Tink. Med. J. Aust. 1: 40, 1958.

2. Trey C., Burns D.G., Saunders S.J.: New Eng. J. Med. 274: 473, 1966.

3. Burnell J.M., Dawborn J.K., Epstein R.B., Gutman R., Thomas $E_{1}$ and Volwiwler $W$.: New Engl. J. Med. 276: 935, 1967.

4. Gonzales G.. Horwitz 1,, Sepúlveda H., Danis O.: Pediatría. 12: 245, 1969.

5. Zacarias J., Brink P., Garcia Huidobro J.: Am. J. Dis. Child. 122: 229, 1971.

6. Redeker A., Yamahiro H.: Lancet. 1: 2. 1973.

7. Sherlock S., Jones E.: Lancet. 2: 169, 1967.

8. Sherlock S.: Postgrad Med. 47: 493, 1971.

9. Zarchy T.: Clinical Proccedins George Washington University, School of Medicine. Vol. 29, No $1,1973$.
10. Marks M.Y., Mauer M., Goldman H.: J. Pediat. 75: 418, 1967.

11. Thompson E., Cwadery J.: Arch. Dis. Child. 43: $368,1968$.

12. McDonald A.: I. Pediat. 63: 916, 1963.

13. Phibbs R.H., Sproul A.: Pediatrics 38: 927, 1966 (Letter).

14. Berger $R$., Liversage R., Chalmets $T$, Graham I., McGoldrick D., Stohlman F.: New Eng1. J. Med. 274: 497, 1966.

15. Adam R.D., Foley I.M. The neurological disorder associated with liver disease in: Metabolic and Toxic disease of the nervotus system. Baltimore, Williams \& Wilkins Co., 1953. Vol, 32.

16. Katz R., Velasco $M_{*}$, Klinger I., Alessandri $H$. Gastroenteterology. 42: 258, 1962.

17. Departamento de Pediatría. Area Norte. Hospital Roberto del Río. Normas de Atención Médica IDtrahospitalaria, 1974. 